\title{
Lichiardopol's conjecture on disjoint cycles in tournaments
}

\author{
Fuhong $\mathrm{Ma}^{*}$ \\ School of Mathematics \\ Shandong University \\ Jinan 250100 China \\ mafuhongsdnu@163.com
}

\author{
Douglas B. West ${ }^{\dagger}$ \\ Departments of Mathematics, \\ Zhejiang Normal University and University of Illinois \\ Jinhua 321004 China and Urbana IL 61801 U.S.A. \\ dwest@math . uiuc . edu \\ Jin Yan $^{\ddagger}$ \\ School of Mathematics \\ Shandong University \\ Jinan 250100 China \\ yanj@sdu.edu.cn
}

Submitted: Mar 9, 2018; Accepted: May 13, 2020; Published: Jun 12, 2020

(c) The authors. Released under the CC BY-ND license (International 4.0).

\begin{abstract}
In 2010, Lichiardopol conjectured for $q \geqslant 3$ and $k \geqslant 1$ that any tournament with minimum out-degree at least $(q-1) k-1$ contains $k$ disjoint cycles of length $q$. Previously the conjecture was known to hold for $q \leqslant 4$. We prove that it holds for $q \geqslant 5$, thereby completing the proof of the conjecture.
\end{abstract}

Mathematics Subject Classifications: 05C70, 05C38

\section{Introduction}

A famous conjecture of Bermond and Thomassen [5] asserts that a digraph (directed graph) with large minimum outdegree must have many disjoint cycles.

Conjecture $1.1([5])$. If a digraph $D$ has minimum outdegree at least $2 k-1$, then $D$ contains $k$ disjoint cycles.

*The first and third authors are supported by National Natural Science Foundation of China grants NNSFC 11671232.

†Supported by National Natural Science Foundation of China grants NNSFC 11871439 and 11971439.

${ }^{\ddagger}$ Corresponding author. 
The conjecture is trivial for $k=1$. It was proved for $k=2$ by Thomassen [25] and for $k=3$ by Lichiardopol, Pór, and Sereni [18]. Instead of studying special values of $k$, one can seek general bounds on the minimum outdegree needed to guarantee $k$ disjoint cycles. Alon [1] showed that $64 k$ suffices, and later Bucić [7] reduced this to $18 k$.

Although Conjecture 1.1 remains open for general digraphs, Bang-Jensen, Bessy, and Thomassé [3] proved it for the special case of tournaments. A tournament is a digraph obtained from a complete graph by assigning a direction to each edge. Since the conjecture holds for tournaments, one can seek stronger conclusions in that case. Lichiardopol [19] conjectured that in a tournament with large minimum outdegree, one can control not only the number of disjoint cycles but also their length. The length of a cycle is the number of edges (and vertices), and a $q$-cycle is a cycle of length $q$.

Conjecture 1.2 ([19]). If $q \geqslant 3$ and $k \geqslant 1$, then every tournament with minimum outdegree at least $(q-1) k-1$ contains $k$ disjoint $q$-cycles.

In a tournament, disjoint cycles of any length lead to disjoint 3-cycles by using chords. Hence the result of [3] yields the special case of Conjecture 1.2 for $q=3$.

Theorem 1.3 ([3]). Every tournament $T$ with minimum outdegree at least $2 k-1$ has $k$ disjoint 3-cycles.

Bessy, Lichiardopol, and Sereni [6] had earlier proved that every tournament with minimum indegree and outdegree both at least $2 k-1$ has $k$ disjoint cycles. In support of Conjecture 1.2, Lichiardopol [19] proved the two weaker theorems below, the first of which improves the result of [6].

Theorem 1.4 ([19]). If $q \geqslant 3$ and $k \geqslant 1$, then every tournament with minimum outdegree and indegree both at least $(q-1) k-1$ contains $k$ disjoint $q$-cycles.

Ma and Yan [20] improved Theorem 1.4 by guaranteeing more than $k$ disjoint cycles under the same conditions, so the conclusion of Theorem 1.4 is not sharp ([21] addressed the special case of 4-cycles in regular tournaments).

Theorem 1.5 ([19]). If $q \geqslant 3$ and $k \geqslant 1$, then every tournament with minimum outdegree at least $(q-1) k-1$ contains $\left\lceil k-1-\frac{k-2}{q}\right\rceil$ disjoint q-cycles.

The case $q=4$ of Conjecture 1.2 was proved in the masters thesis of Zhu [30]. Hence our result in this paper completes the proof of Conjecture 1.2. Using some of our lemmas and similar methods, Wang, Ma, and Yan [28] independently proved the cases $q \leqslant 9$. Our result below for $q \geqslant 5$ is self-contained and does not use any of their arguments.

Theorem 1.6. For $q \geqslant 5$ and $k \geqslant 1$, every tournament with minimum outdegree at least $(q-1) k-1$ contains $k$ disjoint $q$-cycles.

For Conjecture 1.2 (and Theorem 1.6), the degree hypothesis is not known to be sharp. A trivial lower bound on the minimum outdegree needed to guarantee the conclusion is $q k / 2$, since a tournament on $q k-1$ vertices in which every vertex has outdegree $q k / 2-1$ 
does not have enough vertices to have $k$ disjoint $q$-cycles. Given that the needed inequalities are easier to satisfy when $q$ is large, we ask whether there is a positive constant $\varepsilon$ such that minimum outdegree $(1-\varepsilon) q k$ suffices when $q$ is sufficiently large.

The results on tournaments suggest the question of whether large minimum outdegree can guarantee disjoint cycles of the same length in general digraphs, even without constraining which length it is. Thomassen [25] conjectured such a relationship, but Alon [1] showed that it cannot hold. Note that disjoint cycles must also be edge-disjoint.

Theorem $1.7([1])$. For all $r \in \mathbb{N}$, some digraph with minimum outdegree $r$ has no two edge-disjoint cycles of the same length.

Other papers on disjoint cycles in digraphs include [2] and [9]. Results on disjoint cycles are more plentiful for undirected graphs. The Bermond-Thomassen Conjecture is in fact the directed analogue of the Corrádi-Hajnal Theorem [12].

Theorem 1.8 ([12]). For $n, k \in \mathbb{N}$ with $n \geqslant 3 k$, every $n$-vertex undirected graph with minimum degree at least $2 k$ contains $k$ disjoint cycles.

There are many extensions and variations on this result. Those most similar to our work consider the lengths of disjoint cycles guaranteed by a threshold on the minimum degree. Let $\delta(G)$ denote the minimum degree of a graph $G$; also, the order of a graph or digraph is the number of vertices.

Thomassen [26] proved for $k \geqslant 2$ that every graph $G$ with $\delta(G) \geqslant 3 k+1$ and order at least some constant $c_{k}$ contains $k$ disjoint cycles of the same length. Thomassen conjectured that minimum degree $2 k$ suffices, which had earlier been conjectured for $k=2$ by Häggkvist. Egawa [13] proved Thomassen's conjecture for $k \geqslant 3$ with a threshold of $|V(G)| \geqslant 17 k+o(k)$. Verstraëte [27] later proved Thomassen's conjecture in full. Verstraëte also conjectured that order at least $4 k$ is enough to guarantee $k$ disjoint cycles of the same length when $\delta(G) \geqslant 2 k$.

Chiba, Fujita, Kawarabayashi, and Sakuma [10] guaranteed for $k \in \mathbb{N}$ a constant $c_{k}$ such that every graph with order at least $c_{k}$ and minimum degree at least $2 k$ contains $k$ disjoint even cycles, with special exceptions. Other degree conditions for disjoint cycles in undirected graphs can be found in $[4,15,16,22,29]$ and in the survey [11].

\section{Structure of the Proof}

We prove Theorem 1.6 by proving a theorem mainly inspired by the proof of the BermondThomassen Conjecture for tournaments by Bang-Jensen, Bessy, and Thomassé [3].

Theorem 2.1. Fix $k, q \in \mathbb{N}$ with $q \geqslant 5$ and $k \geqslant 2$, and let $T$ be a tournament with minimum outdegree at least $(q-1) k-1$. For any family $\mathcal{F}$ of $k-1$ disjoint $q$-cycles in $T$, there is a family of $k$ disjoint $q$-cycles in $T$ using at most $3 q-6$ vertices outside the cycles in $\mathcal{F}$. 
Our first task is to show that Theorem 2.1 suffices. We use the notation $\left\langle v_{1}, \ldots, v_{q}\right\rangle$ for a path with vertices $v_{1}, \ldots, v_{q}$ in order (the edges are $\left\{v_{i} v_{i+1}: 1 \leqslant i<q\right\}$ ); we call this a $v_{1}, v_{q}$-path. We use the notation $\left[v_{1}, \ldots, v_{q}\right]$ for a cycle with vertices $v_{1}, \ldots, v_{q}$ in order; the edges are those of $\left\langle v_{1}, \ldots, v_{q}\right\rangle$ plus $v_{q} v_{1}$. The outdegree of a vertex $v$ is $d^{+}(v)$, and the minimum outdegree in a digraph $D$ is $\delta^{+}(D)$.

It is well-known that every tournament contains a spanning path (Rédei's Theorem [24]), and that a tournament is strongly connected if and only if it contains a cycle through all the vertices (Camion's Theorem [8]), where a digraph is strongly connected or strong if it contains a $u, v$-path for any two vertices $u$ and $v$. Also, a strong tournament (or the subtournament induced by any cycle) is pancyclic, meaning that it has cycles of all lengths from 3 through the number of vertices. We say "by pancyclicity" to invoke this. Finally, Moon [23] showed that every strong tournament with at least three vertices is vertex pancyclic, meaning that through any vertex there are cycles of all lengths.

Lemma 2.2. Theorem 2.1 implies Theorem 1.6.

Proof. Assuming that Theorem 2.1 holds, we prove Theorem 1.6 by induction on $k$. When $k=1$, we are given a tournament $T$ with $\delta^{+}(T) \geqslant q-2$. Since every tournament has a spanning path, we may let $\left\langle v_{1}, \ldots, v_{n}\right\rangle$ be a spanning path of $T$. Since $d^{+}\left(v_{n}\right) \geqslant q-2$ and $v_{n-1}$ is not an outneighbor of $v_{n}$, the edge to the earliest outneighbor of $v_{n}$ along $P$ completes a cycle of length at least $q$ in $T$. By pancyclicity, $T$ contains a $q$-cycle.

For the induction step, suppose $k>1$. We have $\delta^{+}(T) \geqslant(q-1) k-1>(q-1)(k-1)-1$. By the induction hypothesis, $T$ contains $k-1$ disjoint $q$-cycles. From this family $\mathcal{F}$ of $k-1$ disjoint $q$-cycles, Theorem 2.1 produces a family of $k$ disjoint $q$-cycles.

We will prove Theorem 2.1 by considering two cases. In Theorem 4.1, we will prove it for $q \geqslant 5$ and $k \leqslant q$. In Theorem 5.1, we will prove by induction on $k$ that the conclusion holds when $q \geqslant 5$ and $k>q$, using Theorem 4.1 as a basis. The restriction to using $3 q-6$ vertices outside $\mathcal{F}$ will be used in this inductive proof.

\section{Cycles and Paths in Tournaments}

Here we prove some structural lemmas about tournaments that will be useful in the proof of Theorem 4.1. We use $T[S]$ to denote the subtournament of $T$ induced by the vertex set $S$. We also let $d^{+}(X, Y)$ denote the number of edges with tail in $X$ and head in $Y$, where $X$ and $Y$ may be a set of vertices or a single vertex. When $u v$ is an edge, we say that $v$ is a successor of $u$ and $u$ is a predecessor of $v$.

Lemma 3.1. If $C$ is a cycle of length $m$ in a tournament $T$, where $m \geqslant 4$, then $T[V(C)]$ contains a cycle $C^{\prime}$ of length $m-1$ such that the omitted vertex $u$ of $C$ has at least two predecessors in $V\left(C^{\prime}\right)$.

Proof. Since every strong tournament is pancyclic, $T[V(C)]$ contains a cycle $C^{*}$ of length $m-1$. Let $u^{\prime}$ be the vertex of $C$ not in $C^{*}$, and let $v$ be the predecessor of $u^{\prime}$ on $C$. If $u^{\prime}$ has another predecessor on $C^{*}$, then let $u=u^{\prime}$ and $C^{\prime}=C^{*}$. Otherwise, let $\langle v, w, x\rangle$ be 
the portion of $C^{*}$ leaving $v$ (this exists since $m \geqslant 4$ ). Since $u^{\prime}$ has only one predecessor in $V(C)$, both $u^{\prime} w$ and $u^{\prime} x$ are edges. Now form $C^{\prime}$ by replacing $w$ with $u^{\prime}$ in $C^{*}$, and let $u=w$. The omitted vertex $u$ now has predecessors $v$ and $u^{\prime}$ on $C^{\prime}$.

We believe that for $m \geqslant 2 r$, a strong tournament with $m$ vertices contains a cycle of length $m-1$ such that the omitted vertex has at least $r$ predecessors in $C^{\prime}$. We proved this for $r=3$, but the proof is much longer than for $r=2$, and we only need the result for $r=2$. The threshold on $m$ is sharp; a tournament on $2 r-1$ vertices in which every vertex has $r-1$ predecessors and $r-1$ successors has no vertex with $r$ predecessors.

Lemma 3.2. If $C$ is a cycle of length $m$ in a tournament $T$, where $m \geqslant 5$, then $T[V(C)]$ contains a cycle $C^{\prime \prime}$ of length $m-2$ omitting vertices $x$ and $y$ with $x y \in E(T)$ such that $y$ has at least one predecessor in $V\left(C^{\prime \prime}\right)$.

Proof. We use Lemma 3.1 twice. First, it gives us a cycle $C^{\prime}$ in $T[V(C)]$, with length $m-1$, such that the remaining vertex $u$ has at least two predecessors in $V\left(C^{\prime}\right)$. We then apply Lemma 3.1 using this $C^{\prime}$ as $C$; it gives us a cycle $C^{\prime \prime}$ in $T\left[V\left(C^{\prime}\right)\right]$, with length $m-2$, such that the remaining vertex $v$ has at least two predecessors in $V\left(C^{\prime \prime}\right)$. From $C$, the vertices $u$ and $v$ have been omitted. Each has at least one predecessor in $V\left(C^{\prime \prime}\right)$. Hence we let $x$ be the tail and $y$ be the head of the edge joining $u$ and $v$.

We say that a vertex set $X$ dominates a vertex set $Y$ in a tournament if every edge joining $X$ and $Y$ is oriented from $X$ to $Y$.

Lemma 3.3. If $C$ is a cycle of length $m$ in a tournament $T$, where $m \geqslant 4$, and $S$ is a 3 -set in $V(C)$, then at least one vertex of $S$ has at least two predecessors in $V(C)$.

Proof. If the claim fails, then $T[S]$ is a 3-cycle. Now failure of the claim requires $S$ to dominate $V(C)-S$, contradicting that $C$ is a cycle.

Lemma 3.4. Let $T$ be a tournament with minimum outdegree at least $(q-1) k-1$, where $2 \leqslant k \leqslant q$ and $q \geqslant 5$. Let $\mathcal{F}$ be a family of $k-1$ disjoint $q$-cycles in $T$, with vertex sets $V_{1}, \ldots, V_{k-1}$ inducing cycles $C_{1}, \ldots, C_{k-1}$. If $S_{1}$ and $S_{2}$ partition the remaining vertices (outside $\bigcup V_{i}$ ), with $S_{2}$ dominating $S_{1}$ in $T$ and $\left|S_{1}\right| \leqslant q$, then

(a) $d^{+}\left(V^{*}, S_{1}\right) \leqslant \frac{\left|S_{1}\right|+1}{2 q-2}\left|S_{1}\right|$ for some $V^{*} \in\left\{V_{1}, \ldots, V_{k-1}\right\}$.

(b) If $\left|S_{1}\right| \geqslant 2$, then $d^{+}\left(S_{1}, z\right) \geqslant 2$ for any $z \in V^{*}$.

(c) If $\left|S_{1}\right| \leqslant q-1$, then $d^{+}\left(u, V^{*}\right) \geqslant 3$ for any $u \in S_{1}$.

(d) If $S_{1}$ dominates $z \in V^{*}$, and $z$ has at least $r$ predecessors in $V^{*}$, then $d^{+}\left(z, S_{2}\right) \geqslant r$.

Proof. of (a): Since $S_{2}$ dominates $S_{1}$,

$d^{+}\left(S_{1}, \bigcup V_{i}\right) \geqslant[(q-1) k-1]\left|S_{1}\right|-\frac{\left|S_{1}\right|\left(\left|S_{1}\right|-1\right)}{2}=\left[(q-1)(k-1)+\frac{2 q-\left|S_{1}\right|-3}{2}\right]\left|S_{1}\right|$.

By the pigeonhole principle, we have $C_{i} \in \mathcal{F}$ with $d^{+}\left(S_{1}, V_{i}\right) \geqslant\left(q-1+\frac{2 q-\left|S_{1}\right|-3}{2(k-1)}\right)\left|S_{1}\right|$. Since $\left|S_{1}\right| \leqslant q$ and $q \geqslant 3$, we have $2 q-\left|S_{1}\right|-3 \geqslant 0$. With this and $k \leqslant q$, we obtain $d^{+}\left(S_{1}, V_{i}\right) \geqslant\left(q-\frac{\left|S_{1}\right|+1}{2 q-2}\right)\left|S_{1}\right|$. Let $C^{*}$ be this cycle $C_{i}$, with vertex set $V^{*}$. 
Proof of $(b)$ : If $d^{+}\left(S_{1}, z\right) \leqslant 1$ for some $z \in V^{*}$, then $d^{+}\left(V^{*}, S_{1}\right) \geqslant d^{+}\left(z, S_{1}\right) \geqslant\left|S_{1}\right|-1$. Using part (a), we obtain

$$
\left|S_{1}\right|-1 \leqslant \frac{\left|S_{1}\right|+1}{2 q-2}\left|S_{1}\right| .
$$

With $s=\left|S_{1}\right|$, the inequality can be rewritten as $2 q-2-s(2 q-3-s) \geqslant 0$. However, with $2 \leqslant s \leqslant q$, the left side of this is negative when $q \geqslant 5$.

Proof of $(c)$ : If $d^{+}\left(u, V^{*}\right) \leqslant 2$ for some $u \in S_{1}$. Now $d^{+}\left(V^{*}, S_{1}\right) \geqslant d^{+}\left(V^{*}, u\right) \geqslant q-2$. Using (a) and $\left|S_{1}\right| \leqslant q-1$, we obtain $q-2 \leqslant \frac{q}{2 q-2}(q-1)$, which requires $q \leqslant 4$.

Proof of $(d)$ : Since $z$ is dominated by $S_{1}$ and has at least $r$ predecessors in $V^{*}$,

$$
d^{+}\left(z, S_{2}\right)=d^{+}(z)-d^{+}\left(z, \bigcup_{i} V_{i}\right) \geqslant(q-1) k-1-[q(k-1)-(r+1)]=q-k+r .
$$

Since $k \leqslant q$, we obtain $d^{+}\left(z, S_{2}\right) \geqslant r$.

\section{The Case of Small $k$}

In this section we prove Theorem 2.1 for $k \leqslant q$, stated as Theorem 4.1. We constructively produce the desired family $\mathcal{F}^{*}$ of $k$ disjoint $q$-cycles by iteratively increasing the length of a cycle found in the subtournament $T^{\prime}$ obtain by deleting the vertices in the given $k-1$ disjoint $q$-cycles. The subtournament $T^{\prime}$ has a spanning path $P$; let $v$ be its last vertex. If $v$ lies in a cycle of length at least $q$ in $T^{\prime}$, then by pancyclicity $T^{\prime}$ has a $q$-cycle and we are done. Hence our approach, given a longest cycle through $v$ in $T^{\prime}$ (in the first step the length may be 0 ), is to rearrange $\mathcal{F}$ to find a new family $\mathcal{F}^{\prime}$ of $k-1$ disjoint $q$-cycles so that the vertex at the end of the resulting remaining path $P^{\prime}$ lies in a longer cycle.

Some claims in this argument are not valid when $q=4$. Nevertheless, the same framework applies when $q=4$, with additional more detailed reasoning.

Theorem 4.1. Given $k, q \in \mathbb{N}$ with $q \geqslant 5$ and $k \leqslant q$, let $T$ be a tournament with $\delta^{+}(T) \geqslant(q-1) k-1$. For any family $\mathcal{F}$ of $k-1$ disjoint $q$-cycles in $T$, there is a family $\mathcal{F}^{*}$ of $k$ disjoint $q$-cycles in $T$ using at most $3 q-6$ vertices outside the cycles in $\mathcal{F}$.

Proof. These hypotheses are the same as those of Lemma 3.4 once we obtain a partition $\left(S_{2}, S_{1}\right)$ of the vertices outside $\mathcal{F}$ such that $S_{2}$ dominates $S_{1}$. Given such a partition, let $C^{*}$ with vertex set $V^{*}$ be the cycle in $\mathcal{F}$ guaranteed by Lemma 3.4(a). Let $P$ be a spanning path through the subtournament $T^{\prime}$ of vertices outside $\mathcal{F}$, with last vertex $v$.

When $T^{\prime}$ has a cycle through $v$, let $l$ be the maximum length of such a cycle; otherwise $l=0$. If $l \geqslant q$, then pancyclicity of the subtournament spanned by this cycle provides a $q$-cycle to complete $\mathcal{F}^{*}$. Otherwise, we obtain a new family $\mathcal{F}^{\prime}$ where $l$ is larger, generally by replacing $C^{*}$ with a new cycle $\widehat{C}$ and defining a new path $P^{\prime}$ through the vertices outside $\mathcal{F}^{\prime}$. We will use at most two new vertices in $\widehat{C}$ at each step that increases $l$, except that the steps to reach $l \geqslant 4$ will use at most three new vertices. In addition, 
when a sufficiently long cycle appears, it uses at most $q-1$ new vertices, because the subtournament that was outside the $k-1$ given $q$-cycles entering that step did not contain a cycle of length at least $q$. Thus in total at most $3 q-6$ new vertices are used.

Case 1: $l=0$, so $T^{\prime}$ has no cycle through $v$. We seek $\widehat{C}$ and $P^{\prime}$ so that there is a cycle outside $\mathcal{F}^{\prime}$ through the last vertex of $P^{\prime}$. Let $u$ be the predecessor of $v$ on $P$.

Case 1a. $T^{\prime}$ has no cycle through $u$ (see Figure 1). Let $S_{1}=\{u, v\}$ and $S_{2}=$ $V\left(T^{\prime}\right)-S_{1}$. Since $T^{\prime}$ has no cycle through $u$ or $v, S_{2}$ dominates $S_{1}$. By Lemma 3.4(a), there exists $C^{*} \in \mathcal{F}$ such that $d^{+}\left(V^{*}, S_{1}\right) \leqslant \frac{3}{2 q-2} \cdot 2$, and $\frac{3}{2 q-2} \cdot 2<1$ when $q \geqslant 5$. Thus $S_{1}$ dominates $V^{*}$.

Since $q \geqslant 5$, Lemma 3.2 implies that $T\left[V^{*}\right]$ contains a cycle $C^{\prime \prime}$ of length $q-2$ such that $y$ has a predecessor in $V\left(C^{\prime \prime}\right)$, where $x y$ is the edge in $T\left[V^{*}\right]-V\left(C^{\prime \prime}\right)$. By applying Lemma 3.3 to the $q$-cycle $C^{*}$, among any three vertices in $C^{\prime \prime}$ is a vertex $z$ with at least two predecessors in $V^{*}$. Since also $z$ is dominated by $S_{1}$, Lemma 3.4(d) guarantees $d^{+}\left(z, S_{2}\right) \geqslant 2$. Let $w$ be a successor of $z$ in $S_{2}$, and let $z^{\prime}$ be the successor of $z$ on $C^{\prime \prime}$. Replacing $z z^{\prime}$ in $C^{\prime \prime}$ with $\left\langle z, w, u, z^{\prime}\right\rangle$ yields a $q$-cycle $\widehat{C}$ with two vertices outside $\mathcal{F}$. Replace $C^{*}$ with $\widehat{C}$ to form $\mathcal{F}^{\prime}$ from $\mathcal{F}$.

Since $S_{2}$ dominates $S_{1}$, we can form the $P^{\prime}$ outside $\mathcal{F}^{\prime}$ by appending $v, x, y$ in order to a spanning path of $T\left[S_{2}\right]-w$. Since $S_{1}$ dominates $V^{*}$, and $y$ has at least $x$ and a vertex of $C^{\prime \prime}$ as predecessors in $V^{*}$, Lemma $3.4(\mathrm{~d})$ yields $d^{+}\left(y, S_{2}\right) \geqslant 2$. Thus $y$ has a successor in $S_{2}$ other than $w$, so there is a cycle through $y$ using vertices of $P^{\prime}$.

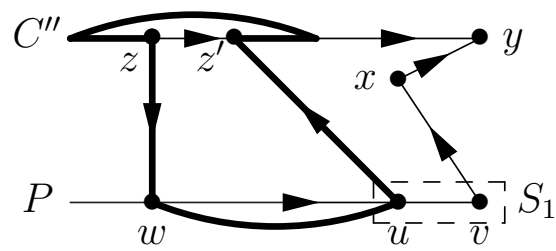

Figure 1: Case 1a of Theorem 4.1.

Case 1b. $T^{\prime}$ has a cycle containing $u$. Let $B$ be a longest cycle containing $u$ in $T^{\prime}$. Let $S_{1}=V(B) \cup\{v\}$ and $S_{2}=V\left(T^{\prime}\right)-S_{1}$. Any edge from $V(B)$ to $S_{2}$ yields a larger strong tournament in $T^{\prime}$ containing $V(B)$, which contains a larger cycle containing $u$. Hence $S_{2}$ dominates $S_{1}$. Also $|V(B)| \leqslant q-1$, since otherwise we have the $k$ th $q$-cycle. Hence $4 \leqslant\left|S_{1}\right| \leqslant q$, so Lemma 3.4 applies. Choose $C^{*}$ with vertex set $V^{*}$ as given by Lemma 3.4.

We will use the following "degree fact". If $\mathcal{F}^{\prime}$ is a family of $k-1$ disjoint $q$-cycles, and the last vertex $v^{\prime}$ in a spanning path through the remaining vertces $S$ has at least two predecessors used by $\mathcal{F}^{\prime}$, then $v^{\prime}$ has a successor in $S$. The reason, using $k \leqslant q$, is

$$
[(q-1) k-1]-[q(k-1)-2]=q-k+1 \geqslant 1 .
$$

There is then a cycle in $S$ through $v^{\prime}$, as desired.

The degree fact yields $d^{+}\left(V^{*}, v\right) \leqslant 1$; otherwise already $T^{\prime}$ has a cycle through $v$. First suppose $d^{+}\left(V^{*}, v\right)=1$ (see Figure 2). Let $z$ be the predecessor of $v$ in $V^{*}$, and let $y$ be 
the successor of $z$ on $C^{*}$. Form $\mathcal{F}^{\prime}$ by replacing $C^{*}$ with the cycle $\widehat{C}$ obtained from $C^{*}$ by replacing $y$ with $v$ (the successor of $y$ on $C^{*}$ is a successor of $v$ ). Since $4 \leqslant\left|S_{1}\right| \leqslant q$, by Lemma 3.4(b) $y$ has at least two predecessors in $S_{1}$, at least one in $B$ (call this vertex $w)$. Since $S_{2}$ dominates $S_{1}$, we can form $P^{\prime}$ by following $P$ through $S_{2}$, then $B$ from the successor of $w$ on $B$ to $w$, and finally the edge $w y$. Since $v$ and $z$ are predecessors of $y$, the degree fact yields a cycle outside $\mathcal{F}^{\prime}$ through $y$. The only vertex used by $\mathcal{F}^{\prime}$ and not by $\mathcal{F}$ is $v$.

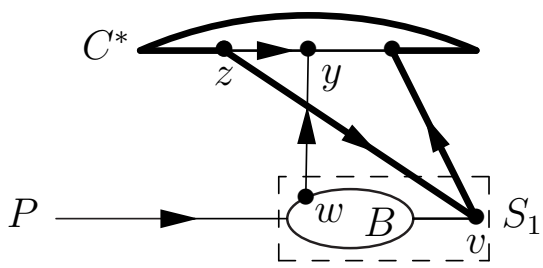

Figure 2: Case 1b of Theorem 4.1 when $d^{+}\left(V^{*}, v\right)=1$.

Therefore, we may assume $d^{+}\left(V^{*}, v\right)=0$, so $v$ dominates $V^{*}$ (see Figures 3 and 4 ). By Lemma 3.1, $T\left[V^{*}\right]$ contains a cycle $C^{\prime}$ of length $q-1$ such that the vertex $y$ of $V^{*}-V\left(C^{\prime}\right)$ has at least two predecessors in $C^{\prime}$. Let $V^{\prime}=V\left(C^{\prime}\right)$.

If $T\left[V^{\prime} \cup\{w\}\right]$ is strong for some $w \in V(B)$, then let $\widehat{C}$ be a spanning cycle in $T\left[V^{\prime} \cup\{w\}\right]$ (outside $\mathcal{F}$ only $w$ is used). Since $V(P)-\{v\}$ dominates $v$, we can let $P^{\prime}$ be a path through all of $V(P)-\{w, v\}$ followed by $v$ and $y$. Since $d^{+}\left(V^{\prime}, y\right) \geqslant 2$, the degree fact applies to $P^{\prime}$.

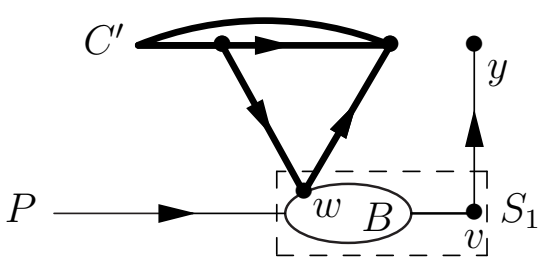

Figure 3: Case $1 \mathrm{~b}$ of Theorem 4.1 when $d^{+}\left(V^{*}, v\right)=0$ and $T\left[V^{\prime} \cup\{w\}\right]$ is strong.

In the remaining case, every vertex of $B$ dominates or is dominated by $V^{\prime}$. If $V^{\prime}$ dominates some vertex of $B$, then $d^{+}\left(V^{*}, S_{1}\right) \geqslant q-1$, but $d^{+}\left(V^{*}, S_{1}\right) \leqslant \frac{\left|S_{1}\right|+1}{2 q-2}\left|S_{1}\right|$ by Lemma 3.4(a). Since $q-1 \leqslant \frac{\left|S_{1}\right|+1}{2 q-2}\left|S_{1}\right| \leqslant \frac{q^{2}+q}{2 q-2}$ requires $q<5$, we conclude that $V(B)$ dominates $V^{\prime}$.

Since also $d^{+}\left(V^{*}, v\right)=0$, now $S_{1}$ dominates $V^{\prime}$ (see Figure 4). We now argue similarly to Case 1a. Since $q-1 \geqslant 4$, by Lemma 3.1 there is a cycle $C^{\prime \prime}$ of length $q-2$ in $T\left[V^{\prime}\right]$ such that the vertex $y^{\prime}$ of $V^{\prime}-V\left(C^{\prime \prime}\right)$ has at least two predecessors in $V\left(C^{\prime \prime}\right)$. Now $y$ and $y^{\prime}$ each have at at least two predecessors in $V^{*}$. Let $z z^{\prime}$ be the edge joining $y$ and $y^{\prime}$.

Since $S_{1}$ dominates $V^{\prime}$, any vertex of $C^{\prime \prime}$ has a successor in $S_{2}$, by Lemma 3.4(d); let $w$ be one such successor. Now $T\left[V\left(C^{\prime \prime}\right) \cup\{w, v\}\right]$ is strong and has a spanning cycle $\widehat{C}$ of length $q$. Form $\mathcal{F}^{\prime}$ from $\mathcal{F}$ by replacing $C^{*}$ with $\widehat{C}$ (note that $\mathcal{F}^{\prime}$ uses only $w$ and $v$ outside $\mathcal{F}$ ). 


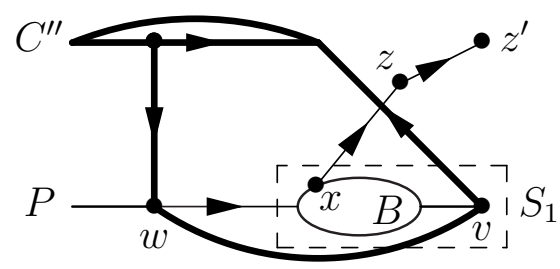

Figure 4: Case $1 \mathrm{~b}$ of Theorem 4.1 when $d^{+}\left(V^{*}, v\right)=0$ and $V(B)$ dominates $V^{\prime}$.

Using Lemma 3.4(b), $d^{+}(V(B), z) \geqslant 1$; let $x$ be a predecessor of $z$ in $V(B)$. Since $S_{2}$ dominates $S_{1}$, we can build a path $P^{\prime}$ that starts with all of $S_{2}-\{w\}$ (in some order), then visits all of $V(B)$ ending with $x$, and finally follows $\left\langle x, z, z^{\prime}\right\rangle$. Since $v$ dominates $V^{*}$, vertex $z^{\prime}$ has at least two predecessors in $\widehat{C}$, and the degree fact applies.

Case 2: $l>0$, so $T^{\prime}$ has a cycle through $v$, the longest having length $l$. Let $C$ be such a cycle of length $l$. We find a new family $\mathcal{F}^{\prime}$ and path $P^{\prime}$ outside it with a longer cycle through the last vertex of $P^{\prime}$. We may assume $l<q$, since otherwise pancyclicity yields the desired $q$-cycle. Let $S_{1}=V(C)$ and $S_{2}=V\left(T^{\prime}\right)-S_{1}$. If there is an edge from $S_{1}$ to $S_{2}$, then $S_{1}$ and part of $P$ induce a strong tournament, which has a longer spanning cycle (containing $v$ ). Hence $S_{2}$ dominates $S_{1}$, and Lemma 3.4 applies.

Let $C^{*}$ with vertex set $V^{*}$ be the cycle in $\mathcal{F}$ guaranteed by Lemma 3.4. Since $q \geqslant 5$, by Lemma $3.2 T\left[V^{*}\right]$ contains a cycle $C^{\prime \prime}$ of length $q-2$ and an edge $x y$ with $\{x, y\}=$ $V^{*}-V\left(C^{\prime \prime}\right)$ such that $y$ has at least one predecessor in $V\left(C^{\prime \prime}\right)$. Let $V^{\prime \prime}=V\left(C^{\prime \prime}\right)$. By Lemma 3.4(a),

$$
d^{+}\left(V^{\prime \prime}, S_{1}\right) \leqslant d^{+}\left(V^{*}, S_{1}\right) \leqslant \frac{\left|S_{1}\right|+1}{2 q-2}\left|S_{1}\right| \leqslant \frac{q}{2 q-2}(q-1)=\frac{q}{2} .
$$

Since $q / 2<q-2$ when $q \geqslant 5$, in $V^{\prime \prime}$ there is a vertex $z$ dominated by $S_{1}$. By Lemma 3.4(d), $z$ has a successor $w$ in $S_{2}$.

Case 2a. $\left|S_{1}\right|=3$ (see Figure 5). Here $d^{+}\left(V^{*}, S_{1}\right) \leqslant \frac{4}{2 q-2} \cdot 3<2$, by Lemma 3.4(a). If $x$ or $y$ has a successor in $S_{1}$, then let $u^{\prime}$ be this successor; otherwise choose $u^{\prime} \in$ $S_{1}$ arbitrarily. Let the cycle $C$ through $S_{1}$ be $\left[u^{\prime}, x^{\prime}, u\right]$, so $x^{\prime} x \in E(T)$. Note that $T\left[V^{\prime \prime} \cup\{w, u\}\right]$ is strong, with a spanning cycle $\widehat{C}$. Replace $C^{*}$ with $\widehat{C}$ in $\mathcal{F}$ to form $\mathcal{F}^{\prime}$.

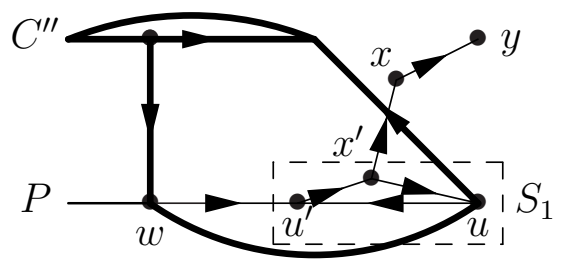

Figure 5: Case 2a of Theorem 4.1.

Let $P^{\prime}$ follow a spanning path through $S_{2}-\{w\}$ and then $\left\langle u^{\prime}, x^{\prime}, x, y\right\rangle$. If $y u^{\prime} \in$ $E(T)$, then we have the cycle $\left[y, u^{\prime}, x^{\prime}, x\right]$ through $y$. Otherwise $d^{+}\left(y, S_{1}\right)=0$, and by 
Lemma 3.4(d) $y$ has at least two successors in $S_{2}$, which means it has one other than $w$. In this case $y$ lies on a cycle of length more than $l$ in $T\left[V\left(P^{\prime}\right)\right]$.

Case 2b. $\left|S_{1}\right| \geqslant 4$ (see Figure 6 ). By pancyclicity, $T\left[S_{1}\right]$ contains a cycle $B$ omitting one vertex $u$ of $S_{1}$. Since $\left|S_{1}\right|=l<q$, Lemma 3.4(c) implies $d^{+}\left(u, V^{*}\right) \geqslant 3$, yielding an edge $u z^{\prime}$ with $z^{\prime} \in V^{\prime \prime}$. Using the edge $z w$ from $V^{\prime \prime}$ to $S_{2}$ (obtained earlier), the path $\left\langle z, w, u, z^{\prime}\right\rangle$ guarantees that $T\left[V^{\prime \prime} \cup\{w, u\}\right]$ is strong and hence has a spanning $q$-cycle $\widehat{C}$. Form $\mathcal{F}^{\prime}$ from $\mathcal{F}$ by replacing $C^{*}$ with $\widehat{C}$.

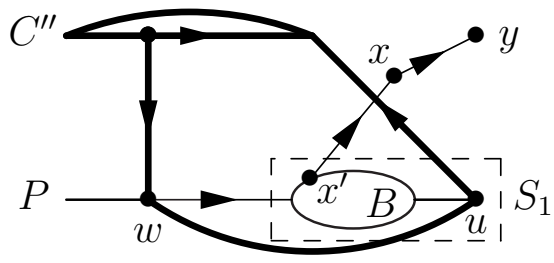

Figure 6: Case 2b of Theorem 4.1 .

By Lemma 3.4(b), $x$ has a predecessor $x^{\prime}$ in $V(B)$. Build the path $P^{\prime}$ outside $\mathcal{F}^{\prime}$ by visiting all of $S_{2}-\{w\}$ (in some order), then all of $V(B)$ ending with $x^{\prime}$, and finally $\left\langle x^{\prime}, x, y\right\rangle$. Since $y$ has at least two predecessors in $V^{\prime \prime} \cup\{x\}$ and hence at most $q-3$ successors in $V^{*}$,

$$
d^{+}\left(y, S_{1} \cup S_{2}\right) \geqslant[(q-1) k-1]-q(k-2)-(q-3)=q-k+2 \geqslant 2 .
$$

If $y$ has a successor outside $\{w, u\}$ in $S_{1} \cup S_{2}$, then with $V(B) \cup\{x, y\}$ it induces a strong tournament of order at least $l+1$, yielding the desired cycle through the last vertex of $P^{\prime}$.

Hence $w$ and $u$ must be the only successors of $y$ in $S_{1} \cup S_{2}$. This forces $d^{+}\left(V^{\prime \prime}, y\right)=1$. If any vertex of $V^{\prime \prime}$ has a successor in $S_{2}$ other than $w$, then we obtain $\mathcal{F}^{\prime}$ as above using that vertex instead of $w$. Hence $w$ must be the only successor of vertices in $V^{\prime \prime}$, so $d^{+}\left(V^{\prime \prime}, S_{2}\right) \leqslant q-2$. We already computed $d^{+}\left(V^{*}, S_{1}\right) \leqslant q / 2$, and one of the edges counted is $y u$, so $d^{+}\left(V^{\prime \prime}, S_{1} \cup S_{2}\right) \leqslant 3 q / 2-3$.

For any $\alpha \in V^{\prime \prime}$, using $k \leqslant q$ we have

$$
d^{+}\left(\alpha, S_{1} \cup S_{2}\right) \geqslant[(q-1) k-1]-q(k-2)-d^{+}\left(\alpha, V^{*}\right) \geqslant q-1-d^{+}\left(\alpha, V^{*}\right) .
$$

When we sum over all $\alpha \in V^{\prime \prime}$, the last term counts all edges in $T\left[V^{\prime \prime}\right]$, possibly $q-2$ edges from $V^{\prime \prime}$ to $x$, and one edge from $V^{\prime \prime}$ to $y$. Hence

$$
d^{+}\left(V^{\prime \prime}, S_{1} \cup S_{2}\right) \geqslant(q-2)(q-1)-\left(\begin{array}{c}
q-2 \\
2
\end{array}\right)-(q-2)-1=(q-2) \frac{q-1}{2}-1 .
$$

The upper and lower bounds on $d^{+}\left(V^{\prime \prime}, S_{1} \cup S_{2}\right)$ require $(q-2)(q-1) / 2-1 \leqslant 3 q / 2-3$, but this inequality requires $q<5$. Hence we obtain the desired improvement $\mathcal{F}^{\prime}$.

In all cases we have improved the family $\mathcal{F}$ as desired. 


\section{The Case of Large $k$}

For the proof of Theorem 5.1, we will use Theorem 4.1 as a basis for induction on $k$. The two theorems have the same conclusion.

Theorem 5.1. Given $k, q \in \mathbb{N}$ with $q \geqslant 5$, let $T$ be a tournament with $\delta^{+}(T) \geqslant(q-1) k-1$. For any family $\mathcal{F}$ of $k-1$ disjoint $q$-cycles in $T$, there is a family of $k$ disjoint $q$-cycles in $T$ whose union has at most $3 q-6$ vertices outside the cycles in $\mathcal{F}$.

We first discuss the basic set-up for the argument, defining notation to be used throughout the proof. We call the desired family an extension of $\mathcal{F}$; finding it is extending $\mathcal{F}$. By Theorem 4.1, we may assume $k \geqslant q+1$. In the tournament $T$, consider a family $\mathcal{F}$ of $k-1$ disjoint $q$-cycles in $T$. We may assume that the tournament $T^{\prime}$ given by deleting the vertices covered by $\mathcal{F}$ contains no cycle with length at least $q$; otherwise we have the desired extension.

Let $P$ be a spanning path in $T^{\prime}$, listed as $\left\langle u_{l}, \ldots, u_{1}\right\rangle$. Since $\delta^{+}(T) \geqslant(q-1) k-1$ implies $|V(T)| \geqslant 2(q-1) k-1$, we have $\left|V\left(T^{\prime}\right)\right| \geqslant 2(q-1) k-1-q(k-1)=(q-2)(k+1)+1$. Since $k \geqslant 3$, we conclude $l=\left|V\left(T^{\prime}\right)\right| \geqslant 4 q-7$.

Partition $V(P)$ into $\left\{U_{1}, S, U_{2}\right\}$ by letting $U_{1}=\left\{u_{1}, \ldots, u_{q}\right\}, S=\left\{u_{q+1}, \ldots, u_{4 q-11}\right\}$, and $U_{2}=V(P)-\left(U_{1} \cup S\right)$. All three sets are nonempty, with $|S|=3 q-11$ and $\left|U_{2}\right| \geqslant 4$. Also, since $T^{\prime}$ contains no $q$-cycle, the edge joining $u_{j}$ and $u_{i}$ is oriented as $u_{j} u_{i}$ when $j-i \geqslant q-1$. Hence $U_{2}$ dominates $U_{1}$.

We aim to find a value $t \in\{1,2\}$ such that we can replace $t$ cycles in $\mathcal{F}$ with $t+1$ cycles of length $q$ using at most $3 q-6$ vertices of $T^{\prime}$. This will complete the proof.

Let $X$ and $Y$ be two disjoint sets of vertices in $T$. We say that there is an $r$-matching from $X$ to $Y$ if the set of edges with tail in $X$ and head in $Y$ contains $r$ edges with no common endpoints. In order to guarantee the existence of desired matchings, we will use the famous König-Egerváry Theorem (König [17], Egerváry [14]), phrased for bipartite digraphs with all edges directed from one part to the other.

Lemma 5.2 ([14, 17]). If there is no r-matching from $X$ to $Y$, then $X \cup Y$ contains a set of at most $r-1$ vertices whose deletion eliminates all edges from $X$ to $Y$.

For the proof of Theorem 5.1, we need a number of additional lemmas. The first is a standard application of the König-Egerváry Theorem, which we will apply with various values of the parameters.

Lemma 5.3. Let $X$ and $Y$ be disjoint vertex sets in $T$, with $s=\min \{|X|,|Y|\}$ and $t=\max \{|X|,|Y|\}$. If $d^{+}(X, Y)>(r-1) t$, where $1 \leqslant r \leqslant s$, then $T$ contains an $r$-matching from $X$ to $Y$.

Proof. Since $r-1$ vertices cover at most $(r-1) t$ edges, the König-Egerváry Theorem implies that the desired matching exists.

Lemma 5.4. Let $C$ be a q-cycle in $\mathcal{F}$. If some vertex $v \in V(C)$ has at least $3 q-6$ successors in $U_{2}$, each with at least two successors in $C$, then there is an extension of $\mathcal{F}$. 
Proof. Let $W \subseteq U_{2}$ be such a set of $3 q-6$ successors of $v$. Since $T[V(C)]$ is strong, by Moon's Theorem it has a $(q-1)$-cycle $C^{\prime}$ containing $v$, omitting one vertex $u$ of $C$. Since each vertex $w \in W$ has a successor in $C$ other than $u$, the subtournament $T\left[V\left(C^{\prime}\right) \cup\{w\}\right]$ is strong and has a spanning $q$-cycle (see Figure 7 ).

Let $T^{\prime}=T-V\left(C^{\prime}\right)$ and $\mathcal{F}_{0}=\mathcal{F}-\{C\}$. Since $T^{\prime}$ omits only $q-1$ vertices, $\delta^{+}\left(T^{\prime}\right) \geqslant$ $(q-1)(k-1)-1$, and $\mathcal{F}_{0}$ is a family of $k-2$ cycles of length $q$ in $T^{\prime}$. Using the induction hypothesis, we can extend $\mathcal{F}_{0}$ to a family $\widehat{\mathcal{F}}$ of $k-1$ cycles of length $q$ in $T^{\prime}$ using at most $3 q-6$ new vertices. Since $|W \cup\{u\}|=3 q-5$, some vertex in $W \cup\{u\}$ is not used by $\widehat{\mathcal{F}}$.

If $u$ is not used, then adding $C$ to $\widehat{\mathcal{F}}$ completes the desired extension. If $u$ is used, then at most $3 q-7$ vertices not in $\mathcal{F}$ are used in $\widehat{\mathcal{F}}$. In this case, some vertex $w \in W$ is not used, and a spanning cycle in $T\left[V\left(C^{\prime}\right) \cup\{w\}\right]$ completes the extension $\mathcal{F}^{\prime}$ using a total of at most $3 q-6$ vertices not in $\mathcal{F}$.

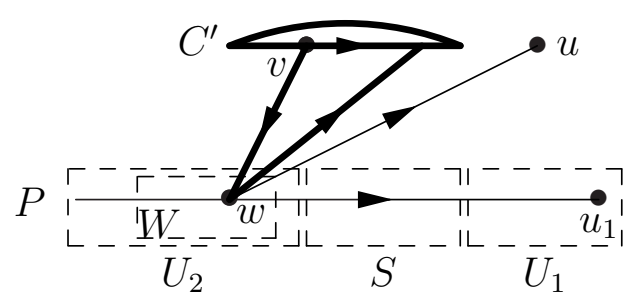

Figure 7: Figure for Lemma 5.4.

The need to find an unused vertex in $W \cup\{u\}$ in the preceding proof is the reason we limit the number of new vertices in Theorem 4.1 and in the induction hypothesis there.

We use Lemma 5.4 to prove the next two lemmas.

Lemma 5.5. Let $C$ be a q-cycle in $\mathcal{F}$. Suppose that $\mathcal{F}$ has no extension.

(i) If $d^{+}\left(C, U_{2}\right) \geqslant 3 q-6$, then $T$ contains a 2-matching from $C$ to $U_{2}$.

(ii) If $d^{+}\left(C, U_{2}\right) \geqslant 6 q-13$, then $T$ contains a 3-matching from $C$ to $U_{2}$.

Proof. (i) If there is no such 2-matching, then by Lemma 5.2 one vertex covers all the edges from $V(C)$ to $U_{2}$. Such a vertex $v$ can only be in $C$, and there is no other edge from $V(C)$ to $U_{2}$. Since $q \geqslant 3$, each of the $3 q-6$ successors of $v$ in $U_{2}$ has at least two successors in $V(C)$. By Lemma 5.4, we have an extension of $\mathcal{F}$.

(ii) If there is no such 3-matching, then by Lemma 5.2 two vertices $u$ and $v$ cover all the edges from $V(C)$ to $U_{2}$, of which there are at least $6 q-13$. If $u$ and $v$ are both in $V(C)$, then one has at least $3 q-6$ successors in $U_{2}$, each of which has at least two successors in $C$, since $q \geqslant 4$. Otherwise, name $u$ and $v$ with $u \in U_{2}$ and $v \in V(C)$; now $v$ has at least $5 q-14$ successors in $U_{2}$ other than $u$. These vertices have no predecessors in $V(C)$ other than $v$; hence they have at least two successors in $V(C)$. Since $5 q-14 \geqslant 3 q-6$ when $q \geqslant 4$, in either case Lemma 5.4 applies to guarantee an extension of $\mathcal{F}$. 
Lemma 5.6. Let $C$ be a q-cycle in $\mathcal{F}$. If $T$ contains

(i) a q-matching from $U_{1}$ to $V(C)$ and a 2-matching from $V(C)$ to $U_{2}$, or

(ii) a $(q-1)$-matching from $U_{1}$ to $V(C)$ and a 3-matching from $V(C)$ to $U_{2}$,

then there is an extension of $\mathcal{F}$.

Proof. We will obtain two disjoint cycles of length at least $q$ in $T[V(C) \cup V(P)]$, where $P$ is a spanning path through the vertices outside $\mathcal{F}$. By pancyclicity, the subtournaments induced by the vertices of these cycles contain $q$-cycles. Since $T^{\prime}$ induces no cycle of length at least $q$, each of these two cycles replacing $C$ uses at most $q-1$ new vertices.

Since $q \geqslant 5$, we have $3 q-11 \geqslant 2 q-6$. Hence $|S| \geqslant 2 q-6$. Since $V(P)$ induces no cycle of length at least $q$, any edge joining two vertices of $P$ with at least $q-2$ vertices between them on $P$ is directed from the earlier to the later vertex.

Let $M$ and $M^{\prime}$ be the given matchings from $U_{1}$ to $V(C)$ and from $V(C)$ to $U_{2}$, respectively. We prove (i) and (ii) together. In either case, let $u$ be the last vertex of $P$ matched into $C$ from $U_{1}$ by $M$; note that $u \in\left\{u_{2}, u_{1}\right\}$. After following the edge from $u$ to $V(C)$, let $v$ be the vertex matched into $U_{2}$ by $M^{\prime}$ that is reached first when continuing along $C$, and let $v w$ be this edge of $M^{\prime}$. Let $Q$ be the path thus followed, from $u$ via $M$, along $C$, ending with $v w$.

Now choose $y z \in M^{\prime}-\{v w\}$ so that $y$ is also the head of an edge in $M$ (under (ii), more than one edge of $M^{\prime}$ remains, but then at most one vertex of $C$ is not covered by $M$.) Say that a vertex of $U_{1}$ leads to $y$ if it is matched by $M$ into the path along $C$ that starts with the successor of $v$ on $C$ and ends at $y$. If $z$ is closer to $S$ than $w$ along $P$, then let $x$ be the highest-indexed vertex of $U_{1}$ (closest to $S$ ) that leads to $y$. Otherwise, let $x$ be the lowest-indexed vertex of of $U_{1}$ that leads to $y$. Let $R$ be the path leaving $x$ via $M$, then along $C$ to $y$, ending with $y z$. We want to form two cycles of length at least $q$ in $T[V(C) \cup V(P)]$ by adding vertices of $P$ to $Q$ or $R$. Let $P[a, b]$ or $C[a, b]$ denote the $a, b$-path along $P$ or $C$.

Case 1: $z$ is closer to $S$ than $w$ along $P$, so $x$ is the highest-indexed vertex of $U_{1}$ leading to $y$ (see Figure 8). First consider $x=u_{q}$. Let one cycle be $R \cup P[z, x]$. Since $|S| \geqslant 2 q-6$ and this cycle contains $S \cup\{x, y, z\}$, it has length at least $2 q-3$, which exceeds $q$. Meanwhile, $P$ has at least $2 q-4$ vertices between $w$ and $u_{q-1}$. Since $2 q-4 \geqslant q-2$, the edge joining them is oriented as $w u_{q-1}$. Hence $Q \cup w u_{q-1} \cup P\left[u_{q-1}, u\right]$ is a cycle with at least $q$ vertices.

When $x \neq u_{q}$, the edge joining $u_{2 q-2}$ and $x$ has the desired orientation, because along $P$ it skips $u_{q}$ and $q-3$ vertices of $S$. Hence $R \cup P\left[z, u_{2 q-2}\right] \cup u_{2 q-2} x$ is a cycle. Since $|S| \geqslant 2 q-6$, it has at least $q-3$ vertices in $S$ plus $\{x, y, z\}$. The other cycle is $Q \cup w u_{2 q-3} \cup P\left[u_{2 q-3}, u_{q+1}\right] \cup u_{q+1} u$. Since $w$ is earlier than $z$ along $P$, there are at least $q-2$ vertices between $w$ and $u_{2 q-3}$ along $P$; the same is true of $u_{q+1}$ and $u$. Hence this is a cycle, and $\left\{u_{2 q-3}, \ldots, u_{q+1}\right\} \cup\{u, v, w\}$ has at least $q$ vertices.

Case 2: $z$ is farther from $S$ than $w$ along $P$, so $x$ is the lowest indexed vertex of $U_{1}$ leading to $y$. Let $x=u_{t}$, and define the paths $Q$ and $R$ as in Case 1 . Note that $t \leqslant q$. We 

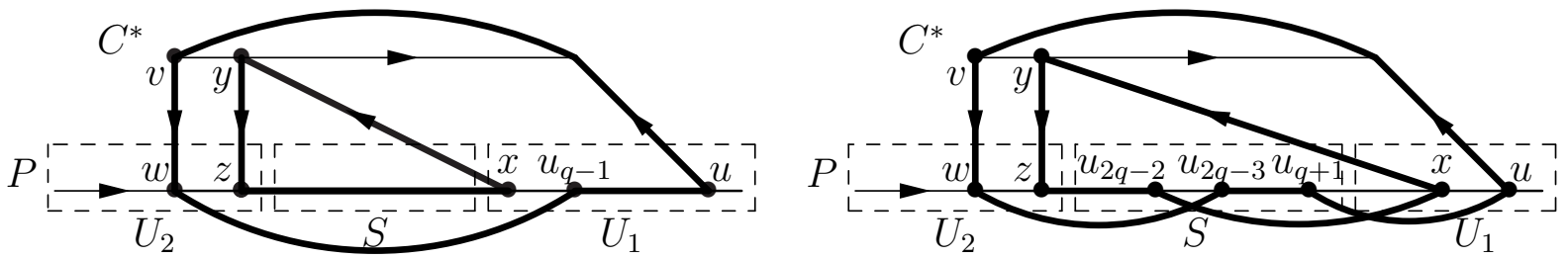

Figure 8: Case 1 of Lemma 5.6.

want the two cycles to be $R \cup z u_{t+q-3} \cup P\left[u_{t+q-3}, x\right]$ and $\left[Q \cup P\left[w, u_{t+q-2}\right] \cup u_{t+q-2} u_{t-1} \cup\right.$ $P\left[u_{t-1}, u\right]$. The jumps along $P$ must skip at least $q-2$ vertices. This is explicit for $u_{t+q-2} u_{t-1}$. Since $(z, w)=\left(u_{j}, u_{i}\right)$ with $j>i>4 q-11$, and $j-(t+q-3) \geqslant 3 q-6-t \geqslant$ $2 q-6$, the other construction is also a cycle if $2 q-6 \geqslant q-1$, which holds when $q \geqslant 5$.

For length at least $q$, the first cycle has $q-3$ vertices along $P$ plus at least $\{x, y, z\}$, and the second adds to $\{u, v, w\}$ all of $S \cup U_{1}$ except the $q-2$ vertices used by the first cycle and $u_{1}$ and maybe $u_{2}$. Since $4 q-11-q \geqslant q-3$ when $q \geqslant 4$, both cycles are long enough.

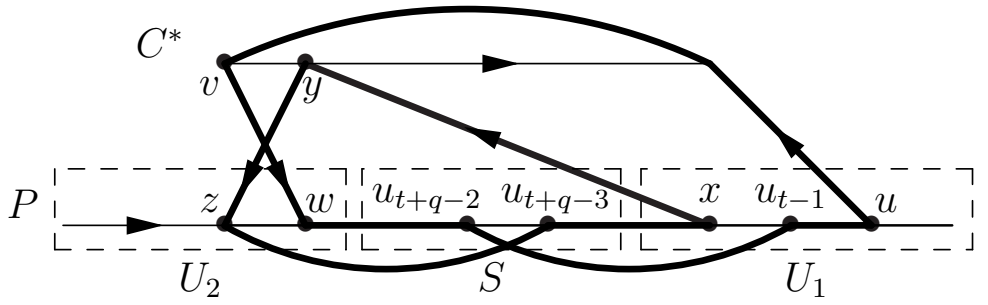

Figure 9: Case 2 of Lemma 5.6.

Lemma 5.7. Let $C$ and $C^{\prime}$ be two members of $\mathcal{F}$, with $W=V(C)$ and $W^{\prime}=V\left(C^{\prime}\right)$. If $T$ contains a q-matching from $U_{1}$ to $W$ and a 3-matching from $W^{\prime}$ to $U_{2}$, and $\left.d^{+}\left(W, W^{\prime}\right)\right) \geqslant$ $q(q-1)+3$, then there is an extension of $\mathcal{F}$.

Proof. Again use the same notation. We may assume that $T[V(P)]$ contains no $q$-cycle. We will extend $\mathcal{F}$ by replacing $C$ and $C^{\prime}$ in $\mathcal{F}$ with three $q$-cycles (except in one case). We must ensure that they introduce at most $3 q-6$ new vertices. The other members of $\mathcal{F}$ remain.

Since $d^{+}\left(W, W^{\prime}\right) \geqslant q(q-1)+3$, the set $W$ has at least three vertices that each dominate $W^{\prime}$ (otherwise, $\left.d^{+}\left(W, W^{\prime}\right) \leqslant 2 q+(q-2)(q-1)=q(q-1)+2\right)$. Label these as $x_{1}, x_{2}, x_{3}$ so that with $w_{1} x_{1}, w_{2} x_{2}, w_{3} x_{3}$ being edges in the $q$-matching from $U_{1}$ to $W$, the vertices $w_{1}, w_{2}, w_{3}$ occur in that order along $P$ through $U_{1}\left(w_{1}\right.$ is closest to $\left.S\right)$. See Figure 10.

Let the edges of the given 3-matching from $W^{\prime}$ to $U_{2}$ be $y_{1} z_{1}, y_{2} z_{2}, y_{3} z_{3}$, indexed so that $z_{3}, z_{1}, z_{2}$ occur in that order along $P\left(z_{2}\right.$ is closest to $\left.S\right)$. Since each vertex in $\left\{x_{1}, x_{2}, x_{3}\right\}$ dominates $W^{\prime}$, we now have three disjoint paths from $U_{1}$ to $U_{2}$; the $i$ th path $R_{i}$ is $\left\langle w_{i}, x_{i}, y_{i}, z_{i}\right\rangle$. 
We complete these three paths to disjoint cycles by adding vertices along the path $P$. Recall that $P$ contains the path $\left\langle u_{4 q-11}, \ldots, u_{q+1}\right\rangle$ through $S$ between $U_{2}$ and $U_{1}$. Along this path of $3 q-11$ vertices define three disjoint paths, each having $q-4$ vertices (one vertex of $S$ is not needed); call them $Q_{2}, Q_{3}, Q_{1}$ in order along $P$.

Let $B_{i}$ be the cycle formed by combining $R_{i}$ and $Q_{i}$; add the edges from the end of each of $R_{i}$ and $Q_{i}$ to the beginning of the other, except that between $z_{2}$ and $Q_{2}$ in $B_{2}$ we follow $P$ to the end of $U_{2}$, and between $Q_{1}$ and $w_{1}$ in $B_{1}$ we follow $P$ through the beginning of $U_{1}$. The edges from $z_{1}$ to $Q_{1}$, from $z_{3}$ to $Q_{3}$, from $Q_{3}$ to $w_{3}$, and from $Q_{2}$ to $w_{2}$, are oriented in the desired direction because they skip at least $q-2$ vertices along $P$ and hence would complete cycles of length at least $q$ if oriented in the other direction.

Note that $B_{3}$ has exactly $q$ vertices. Possibly $B_{1}$ or $B_{2}$ has more vertices due to picking up extras at the beginning of $U_{1}$ or the end of $U_{2}$. However, we can shorten the cycles to length $q$ by omitting vertices at the beginning of $Q_{1}$ and/or the end of $Q_{2}$. This only makes the jumps along $P$ longer, so the edges make the jumps still have the desired orientation. The resulting cycle $B_{i}^{\prime}$ is a $q$-cycle using exactly two vertices used in $\mathcal{F}\left(x_{i}\right.$ and $y_{i}$ ). Hence $\left\{B_{1}^{\prime}, B_{2}^{\prime}, B_{3}\right\}$ replaces $\left\{C, C^{\prime}\right\}$ to yield an extension using $3 q-6$ vertices not used by $\mathcal{F}$.

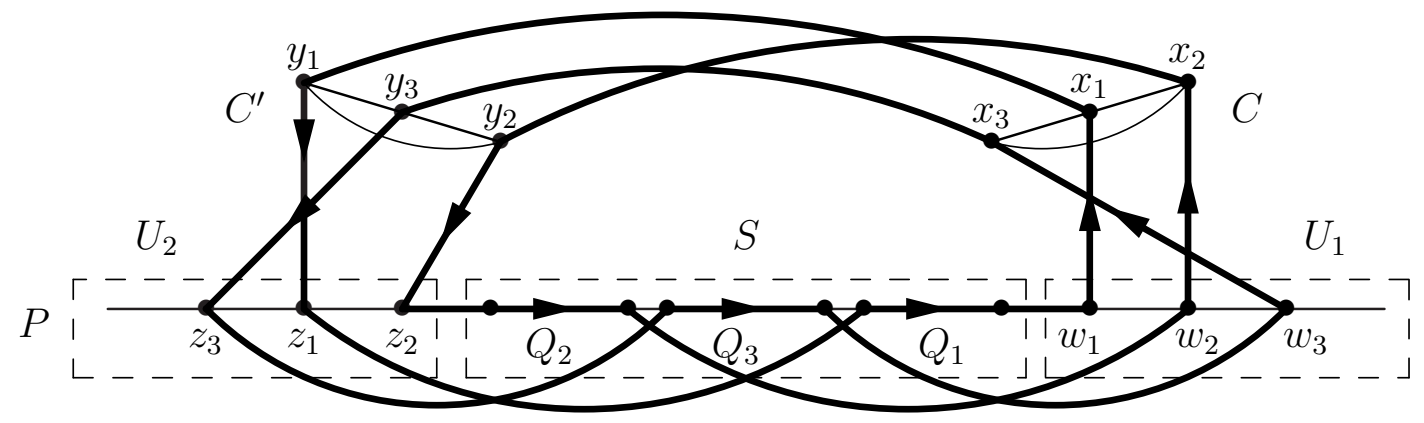

Figure 10: Figure for Lemma 5.7.

Finally we are ready to complete the main proof.

Proof of Theorem 5.1. We will obtain bounds on the sizes of various sets of edges under the assumption that $\mathcal{F}$ has no extension. These will lead to a contradiction.

Let $\mathcal{L}$ consist of the $q$-cycles in $\mathcal{F}$ receiving at least $q(q-1)+1$ edges from $U_{1}$ (thereby guaranteeing a $q$-matching from $U_{1}$, by Lemma 5.3). Let $\mathcal{M}$ consist of the $q$-cycles in $\mathcal{F}$ sending at least $6 q-13$ edges to $U_{2}$ (thereby guaranteeing a 3 -matching to $U_{2}$, by Lemma 5.5(ii)). Let $\mathcal{R}=\mathcal{F}-(\mathcal{L} \cup \mathcal{M})$. Let $l, m$, and $r$, respectively, denote the sizes of $\mathcal{L}, \mathcal{M}$ and $\mathcal{R}$. By Lemma 5.6, $\mathcal{L} \cap \mathcal{M}=\varnothing$. Hence $\{\mathcal{L}, \mathcal{M}, \mathcal{R}\}$ is a partition of $\mathcal{F}$, and

$$
l+m+r=k-1 .
$$

Now consider $d^{+}\left(U_{1}, S \cup U_{2}\right)$. If this is nonzero, then let $u_{q+s}$ be the highest-indexed (earliest) vertex of $P$ having a predecessor in $U_{1}$, and let $u_{q+1-t}$ be the lowest-indexed vertex of $P$ having a successor in $S \cup U_{2}$. Since $P$ gives a path from $u_{q+s}$ to $u_{q+1-t}$, the 
tournament induced by these $s+t$ vertices is strong and has a spanning cycle. Hence $s+t<q$. Also $d^{+}\left(U_{1}, S \cup U_{2}\right) \leqslant s t$. With $s+t \leqslant q-1$, we have $d^{+}\left(U_{1}, S \cup U_{2}\right) \leqslant(q-1)^{2} / 4$.

Next we obtain upper and lower bounds on $d^{+}\left(U_{1}, \mathcal{F}\right)$ in order to obtain an inequality involving $l$ and $m$. Using the computation above,

$$
d^{+}\left(U_{1}, \mathcal{F}\right) \geqslant q[(q-1) k-1]-\left(\begin{array}{l}
q \\
2
\end{array}\right)-\frac{(q-1)^{2}}{4} .
$$

To avoid obtaining an extension of $\mathcal{F}$ via Lemma 5.6, each cycle in $\mathcal{M}$ must avoid a $(q-1)$-matching from $U_{1}$ and hence receives at most $q(q-2)$ edges from $U_{1}$. By definition, each cycle in $\mathcal{L}$ or $\mathcal{R}$ receives at most $q^{2}$ or $q(q-1)$ edges from $U_{1}$, respectively (the latter because otherwise it would be in $\mathcal{L}$ ). Thus

$$
d^{+}\left(U_{1}, \mathcal{F}\right) \leqslant q^{2} l+q(q-2) m+q(q-1) r .
$$

After dividing by $q$, we obtain the following inequality:

$$
(q-1) k-1-\frac{q-1}{2}-\frac{q-2}{4}-\frac{1}{4 q} \leqslant q l+(q-2) m+(q-1) r .
$$

Since $l+m+r=k-1$, we can rewrite the right side as $(q-1)(k-1)+l-m$. Thus

$$
l-m \geqslant \frac{q}{4}-1-\frac{1}{4 q}
$$

and we can drop the $-1 /(4 q)$ term since $l$ and $m$ are integers.

Finally, we will obtain upper and lower bounds on the number of edges leaving $\mathcal{L} \cup \mathcal{R}$ in order to obtain an inequality that cannot be satisfied. First, since $\delta^{+}(T) \geqslant(q-1) k-1$,

$$
d^{+}(\mathcal{L} \cup \mathcal{R}, \overline{\mathcal{L} \cup \mathcal{R}}) \geqslant q(l+r)[(q-1) k-1]-\left(\begin{array}{c}
q(l+r) \\
2
\end{array}\right) .
$$

The absence of extensions imposes bounds on the number of edges leaving $\mathcal{L}$. Since every cycle in $\mathcal{L}$ receives a $q$-matching from $U_{1}$, Lemma 5.5(i) and Lemma 5.6(i) imply $d^{+}\left(\mathcal{L}, U_{2}\right) \leqslant l(3 q-7)$. By Lemma 5.7, $d^{+}(\mathcal{L}, \mathcal{M}) \leqslant \operatorname{lm}[q(q-1)+2]$. Also, $d^{+}(\mathcal{R}, \mathcal{M}) \leqslant$ $q^{2} m r$. Each cycle in $\mathcal{R}$ sends at most $6 q-14$ edges to $\left|U_{2}\right|$ (otherwise it would be placed in $\mathcal{M})$, so $d^{+}\left(\mathcal{R}, U_{2}\right) \leqslant(3 q-7) 2 r$. Also $d^{+}(\mathcal{L} \cup \mathcal{R}, S) \leqslant(3 q-11) q(l+r)$, since $|S|=3 q-11$.

The lower bound $d^{+}\left(U_{1}, \mathcal{F}\right) \geqslant q((q-1) k-1)-\left(\begin{array}{l}q \\ 2\end{array}\right)-(q-1)^{2} / 4$ is from (3). Since every cycle in $\mathcal{M}$ has a 3 -matching to $U_{2}$, by Lemma 5.6 there is no $(q-1)$-matching from $U_{1}$ to a cycle in $\mathcal{M}$; hence $d^{+}\left(U_{1}, \mathcal{M}\right) \leqslant m q(q-2)$. Thus

$$
d^{+}\left(U_{1}, \mathcal{L} \cup \mathcal{R}\right) \geqslant q[(q-1) k-1]-\left(\begin{array}{l}
q \\
2
\end{array}\right)-\frac{(q-1)^{2}}{4}-q(q-2) m .
$$

Let $\alpha=\left(\begin{array}{l}q \\ 2\end{array}\right)+(q-1)^{2} / 4$. Using (2), we conclude

$$
\begin{aligned}
d^{+}\left(\mathcal{L} \cup \mathcal{R}, U_{1}\right) & \leqslant q^{2}(l+r)-q[(q-1) k-1]+q(q-2) m+\alpha \\
& =q^{2}(l+r)-q^{2} k+k q+q+q^{2} m-2 q m+\alpha \\
& =q^{2}(l+r+m-k+1)-q^{2}+k q+q-2 q m+\alpha=q(l+r+2-m-q)+\alpha .
\end{aligned}
$$


Collecting the bounds proved above (with some rearrangement), we have

$$
\begin{aligned}
& d^{+}(\mathcal{L} \cup \mathcal{R}, \overline{\mathcal{L} \cup \mathcal{R}}) \\
& \quad=\quad d^{+}(\mathcal{L} \cup \mathcal{R}, \mathcal{M}) \quad+d^{+}\left(\mathcal{L} \cup \mathcal{R}, U_{2}\right) \quad+d^{+}(\mathcal{L} \cup \mathcal{R}, S) \quad+d^{+}\left(\mathcal{L} \cup \mathcal{R}, U_{1}\right) \\
& \quad \leqslant m\left[q^{2}(l+r)-l(q-2)\right]+(3 q-7)(l+2 r)+(3 q-11) q(l+r)+q(l+r+2-m-q)+\alpha .
\end{aligned}
$$

Combining (5) and (6) and collecting the terms involving $q(l+r)$ yields

$$
\begin{aligned}
q(l+r)\left[(q-1) k-1-\frac{q(l+r)-1}{2}\right. & \left.-m q-3 q+7+\frac{7}{q}\right] \\
& \leqslant-m l(q-2)+(3 q-7) r-q(m+q-2)+\alpha .
\end{aligned}
$$

Using (2), we simplify the last factor on the left:

$$
\begin{aligned}
(q-1) k-1- & \frac{q(l+r)-1}{2}-m q-3 q+7+\frac{7}{q} \\
& =(q-1) k-\frac{q(l+r+m+1)}{2}-\frac{m q}{2}-\frac{5 q}{2}+\frac{13}{2}+\frac{7}{q} \\
& =\left(\frac{q}{2}-1\right)(k-5)-\frac{m q}{2}+\frac{3}{2}+\frac{7}{q} .
\end{aligned}
$$

On the right side of (7), we compute $\alpha-q(q-2)=\frac{-(q-1)(q-3)}{4}+1$. On the left side, we replace $l+r$ with $k-1-m$, and on the right we replace $r$ with $k-1-m-l$. The inequality is now

$$
\begin{aligned}
q(k-1-m) & {\left[\left(\frac{q}{2}-1\right)(k-5)-\frac{m q}{2}+\frac{3}{2}+\frac{7}{q}\right] } \\
& \leqslant-m l(q-2)+(3 q-7)(k-1-m-l)-q m-\frac{(q-1)(q-3)}{4}+1 .
\end{aligned}
$$

The coefficients of $l$ in its only appearances are negative. Hence for given $q, k, m$, the inequality can hold only if it holds when $l$ takes its smallest allowed numerical value. By (4), we have $l \geqslant m-1+(q / 4)$, which yields $l \geqslant m+1$ when $q \geqslant 5$ since $l \in \mathbb{N}$. Setting $l=m+1$, we now have a quadratic inequality for $m$ in terms of $k$ and $q$ :

$$
\begin{aligned}
q(k-1-m) & {\left[\left(\frac{q}{2}-1\right)(k-5)-\frac{m q}{2}+\frac{3}{2}+\frac{7}{q}\right] } \\
& \leqslant-m(m+1)(q-2)+(3 q-7)(k-2 m-2)-q m-\frac{(q-1)(q-3)}{4}+1 .
\end{aligned}
$$

We first collect terms to write this as a quadratic inequality for $m$ :

$$
\left(\frac{q^{2}}{2}+q-2\right) m^{2}+\left[\left(q-q^{2}\right) k+3 q^{2}+\frac{3}{2} q-23\right] m+c \leqslant 0
$$

where $c$ depends only on $k$ and $q$. 
The inequality $l \geqslant m+1$ also yields $k-1=l+m+r \geqslant 2 m+1$, and hence $m \leqslant\lfloor k / 2\rfloor-1$. We thus want to show that (10) cannot be satisfied when $k \geqslant q+1 \geqslant 6$ and $0 \leqslant m \leqslant\lfloor k / 2\rfloor-1$.

To obtain the desired contradiction, it suffices to show that the left side of (10) is positive at its lowest allowed point. Since the coefficient of the quadratic term is positive, the quadratic polynomial is minimized where its derivative is 0 . This occurs when

$$
\left(q^{2}+2 q-4\right) m=\left(q^{2}-q\right) k-\left(3 q^{2}+\frac{3}{2} q-23\right) .
$$

The analysis simplifies if the lowest value of the polynomial in (10) among allowed values for $m$ occurs at the highest allowed value, $\lfloor k / 2\rfloor-1$. Since the graph of a quadratic polynomial is symmetric around the minimum, when $k$ is even this holds if the minimizing point is at least $k / 2-3 / 2$. When $k$ is odd this also suffices, due to the floor function.

Thus we want the solution for $m$ in (11) to be at least $(k-3) / 2$. This holds unless $\left(q^{2}+2 q-4\right) \frac{k-3}{2}>\left(q^{2}-q\right) k-\left(3 q^{2}+\frac{3}{2} q-23\right)$. Solving for $k$ yields

$$
k<\frac{3 q^{2}-3 q-34}{q^{2}-4 q+4} .
$$

The right side of (12) is less than 5 for all $q$ (since $5\left(q^{2}-4 q+4\right)-\left(3 q^{2}-3 q-34\right)$ has no root). Hence in the case $k \geqslant q+1 \geqslant 5$ we have the desired reduction.

Hence it suffices to show that (10) or equivalently (9) cannot hold when $m=\lfloor k / 2\rfloor-1$. When $k$ is even, we set $m=k / 2-1$ in (9). This simplifies the expression, since now $k-2 m-2=0$ and $k-1-m=k / 2$. We require

$$
q \frac{k}{2}\left[\left(\frac{q}{2}-1\right)(k-5)-\frac{(k-2) q}{4}+\frac{3}{2}+\frac{7}{q}\right] \leqslant-\frac{(k-2) k}{4}(q-2)-q \frac{k-2}{2}-\frac{(q-1)(q-3)}{4}+1 .
$$

The right side is negative and decreases as $k$ increases. The coefficient on $k$ in the third factor on the left is $q / 4-1$, which is positive, so the factor increases as $k$ increases. Hence it suffices to show that the inequality cannot hold when $k$ takes its least allowed value, $q+1$. The inequality then simplifies to

$$
q \frac{q+1}{2}\left[\left(\frac{q}{2}-1\right)(q-4)-\frac{(q-1) q}{4}+\frac{3}{2}+\frac{7}{q}\right] \leqslant-(q-1)\left[\frac{(q+1)(q-2)}{4}+\frac{q}{2}+\frac{q-3}{4}\right]+1 .
$$

The left side increases with $q$, and the right side decreases with $q$, so it suffices to show that the inequality fails when $q=4$. The left side is then $10 / 4$ and the right side is $-53 / 4$.

When $k$ is odd, we instead set $m=(k-3) / 2$. In this case $k-2 m-2=1$ and $k-1-m=(k+1) / 2$, so $(9)$ becomes

$$
\begin{aligned}
q \frac{k+1}{2} & {\left[\left(\frac{q}{2}-1\right)(k-5)-\frac{(k-3) q}{4}+\frac{3}{2}+\frac{7}{q}\right] } \\
& \leqslant-\frac{(k-3)(k-1)(q-2)}{4}+(3 q-7)-\frac{(k-3) q}{2}-\frac{(q-1)(q-3)}{4}+1 .
\end{aligned}
$$


Again the last factor on the left increases with $k$, so again it suffices to consider the smallest allowed value for $k$, which is $q+1$. We require

$$
\begin{aligned}
q \frac{q+2}{2} & {\left[\left(\frac{q}{2}-1\right)(q-4)-\frac{(q-2) q}{4}+\frac{3}{2}+\frac{7}{q}\right] } \\
& \leqslant-\frac{(q-2) q(q-2)}{4}+(3 q-7)-\frac{(q-2) q}{2}-\frac{(q-1)(q-3)}{4}+1 .
\end{aligned}
$$

Again the left side increases with $q$ while the right side decreases, so we just show that the inequality cannot hold when $q=4$, where the left side is 15 and the right is $-11 / 4$.

Thus the inequality cannot hold for any allowed values of the parameters, and an extension must exist.

Although the computation at the end of the proof works for $q=4$, other difficulties arise when extending to $q=4$. First, instead of $l \geqslant m+1$ we must also consider $l=m$. Also, although most cases in the proofs of Theorem 4.1 and Lemmas 5.6 and 5.7 extend to $q=4$ (sometimes with additional case analysis), the very special part of Case 2 in Lemma 5.6 when $(u, x, w, z)=\left(u_{2}, u_{4}, u_{6}, u_{7}\right)$ does not work. This can be fixed by changing $|S|$ from $3 q-11$ to $3 q-10$, but then the term $3 / 2$ on the left side of the numerical inequality beomes $1 / 2$, and the desired contradiction fails to occur in the one special case $(q, k, l, m)=(4,5,1,1)$. Further analysis for that case could extend the proof to $q=4$.

\section{References}

[1] N. Alon, Disjoint directed cycles, J. Combinatorial Theory Ser. B, 68(2):167-178, 1996.

[2] Y. Bai, B. Li, H. Li, Vertex-disjoint cycles in bipartite tournaments, Discrete Math., 338:1307-1309, 2015.

[3] J. Bang-Jensen, S. Bessy, S. Thomassé, Disjoint 3-cycles in tournaments: A proof of the Bermond-Thomassen conjecture for tournaments, J. Graph Theory, 75(3):284302, 2014.

[4] J. Bensmail, A. Harutyunyan, N-K. Le, B. Li, N. Lichiardopol, Disjoint cycles of different lengths in graphs and digraphs, Electronic J. Combinatorics, 24(4):\#P4.37, 2017.

[5] J. C. Bermond, C. Thomassen, Cycles in digraphs - a survey, J. Graph Theory, 5(1):1-43, 1981.

[6] S. Bessy, N. Lichiardopol, and J.-S. Sereni, Two proofs of the Bermond-Thomassen Conjecture for tournaments with bounded minimum in-degree, Discrete Math., 310(3):557-560, 2010.

[7] M. Bucić, An improved bound for disjoint directed cycles, Discrete Math., 341(8):2231-2236, 2018.

[8] P. Camion, Chemins et circuits hamiltoniens des graphes complets. C. R. Acad. Sci. Paris, 249:2151-2152, 1959.

[9] G. Chen, R. J. Gould, H. Li, Partitioning vertices of a tournament into independent cycles, J. Combinatorial Theory Ser. B, 83(2):213-220, 2001. 
[10] S. Chiba, S. Fujita, K. Kawarabayashi, T. Sakuma, Minimum degree conditions for vertex-disjoint even cycles in large graphs, Advances in Applied Mathematics, 54:105$120,2014$.

[11] S. Chiba, T. Yamashita, Degree conditions for the existence of vertex-disjoint cycles and paths: a survey, Graphs and Combinatorics, 34:1-83, 2018.

[12] K. Corrádi, A. Hajnal, On the maximal number of independent circuits in a graph, Acta Math. Acad. Sci. Hungar., 14:423-439, 1963.

[13] Y. Egawa, Vertex-disjoint cycles of the same length, J. Combinatorial Theory Ser. B, 66:168-200, 1996.

[14] E. Egerváry, On combinatorial properties of matrices (Hungarian with German summary), Mat. Lapok, 38:16-28, 1931.

[15] Z. Jiao, H. Wang, J. Yan, Disjoint cycles in a graph with distance degree sum conditions, Discrete Math., 340:1203-1209, 2017.

[16] H. A. Kierstead, A. V. Kostochka, E. C. Yeager, On the Corrádi-Hajnal theorem and a question of Dirac, J. Combinatorial Theory Ser. B, 122:121-148, 2017.

[17] D. König, Graphen und Matrizen, Mat. Lapok, 38:116-119, 1931.

[18] N. Lichiardopol, A. Pór, J. S. Sereni, A step toward the Bermond-Thomassen conjecture about disjoint cycles in digraphs, SIAM J. Discrete Math., 23(2):979-992, 2009.

[19] N. Lichiardopol, Vertex-disjoint directed cycles of prescribed length in tournaments with given minimum out-degree and in-degree, Discrete Math., 310(19):2567-2570, 2010.

[20] F. Ma, J. Yan, An improvement of Lichiardopol's theorem on disjoint cycles in tournaments, Appl. Math. Comput., 347:162-168, 2019.

[21] F. Ma, J. Yan, On the number of disjoint 4-cycles in regular tournaments, Discussiones Math. Graph Theory, 38:491-498, 2018.

[22] F. Ma, J. Yan, The confirmation of a conjecture on disjoint cycles in a graph, Discrete Math., 341:2903-2911, 2018.

[23] J. W. Moon, On subtournaments of a tournament, Can. Math. Bull., 9:297-301, 1966.

[24] L. Rédei, Ein kominatischer Satz, Acta. Litt. Szeged, 7:39-43, 1934.

[25] C. Thomassen, Disjoint cycles in digraphs, Combinatorica, 3(3-4):393-396, 1983.

[26] C. Thomassen, Girth in graphs, J. Combinatorial Theory Ser. B, 35(2):129-141, 1983.

[27] J. Verstraëte, Vertex-disjoint cycles of the same length, J. Combinatorial Theory Ser. $B, 88(1): 45-52,2003$.

[28] Y. Wang, F. Ma, and J. Yan, A proof of Lichiardopol conjecture for disjoint cycles in tournaments, manuscript.

[29] J. Yan, S. Zhang, J. Cai, Fan-type condition on disjoint cycles in a graph, Discrete Math., 341:1160-1165, 2018.

[30] S. Zhu, Masters' Thesis, Shandong University, 2019 (Chinese). 